\title{
ON THE CONTROLLABILITY AND STABILIZATION OF THE LINEARIZED BENJAMIN-ONO EQUATION*
}

\author{
Felipe Linares ${ }^{1}$ AND JAime H. ORTEGA ${ }^{2,3}$
}

\begin{abstract}
In this work we are interested in the study of controllability and stabilization of the linearized Benjamin-Ono equation with periodic boundary conditions, which is a generic model for the study of weakly nonlinear waves with nonlocal dispersion. It is well known that the Benjamin-Ono equation has infinite number of conserved quantities, thus we consider only controls acting in the equation such that the volume of the solution is conserved. We study also the stabilization with a feedback law which gives us an exponential decay of the solutions.
\end{abstract}

Mathematics Subject Classification. 37L50, 93B05, 93C20, 93D15.

Received April 18, 2003. Revised March 11 and April 2, 2004.

\section{IntRoduction}

In this work, we consider the linearized Benjamin-Ono (BO) equation in the periodic setting, that is,

$$
u_{t}+\mathcal{H} u_{x x}=0, \quad x \in(-L, L), t>0,
$$

where $\mathcal{H}$ is the Hilbert transform, defined by

$$
\mathcal{H}(f)(x)=\mathrm{PV} \int_{-L}^{L} \cot \frac{\pi(x-y)}{2 L} f(y) \mathrm{d} y .
$$

The Benjamin-Ono equation,

$$
u_{t}+\mathcal{H} u_{x x}+u u_{x}=0
$$

is an integro-differential equation that serves as a generic model for the study of weakly nonlinear long waves incorporating the lowest-order effects of nonlinearity and nonlocal dispersion. In particular, the propagation of internal waves in stratified fluids of great depth is described by the BO equation (see $[3,18]$ ) and turns out to be important in other physical situations as well (see $[9,12,16]$ ). Among noticeable properties of this equation we can mention that it defines a Hamiltonian system, can be solved by an analogue of the inverse scattering

\footnotetext{
Keywords and phrases. exact controllability, stabilization, Benjamin-Ono equation, dispersive equation.

* Partially supported by Grants FONDECYT 1000543 and 7000543.

1 IMPA, Estrada Dona Castorina 110, Rio de Janeiro, 22460-320, Brasil; linares@impa.br

2 Universidad de Chile, Facultad de Ciencias Físicas y Matemáticas. Departamento de Ingeniería Matemática, Casilla 170/3, Correo 3, Santiago, Chile.

3 Departamento de Ciencias Básicas, Universidad del Bío-Bío, Avda. Andrés Bello s/n, Casilla 447, Chillán, Chile; jortega@dim.uchile.cl
} 
method (see [2]), admits (multi)soliton solutions (see [6]) and satisfies infinitely many conserved quantities (see $[6])$, for instance, we have that

$$
\int_{-L}^{L} u(x, t) \mathrm{d} x=\int_{-L}^{L} u_{0}(x) \mathrm{d} x, \quad \forall t>0
$$

Regarding the initial value problem (IVP) associated to the BO equation (3) the periodic and real line settings have been considered. In the periodic case the only result available in the literature is due to Abdelouhab, Bona, Felland and Saut [1]. They obtained a local result for data in $H^{s}(\mathbb{T}), s>3 / 2$. On the other hand, the situation in the real line has been extensively studied. In this case, the Hilbert transform is defined via the Fourier transform as $\widehat{\mathcal{H}(f)}=-i \operatorname{sgn}(\xi) \widehat{f}$. In addition to the previously mentioned work [1], Iório [11], Ponce [21] and more recently Koch and Tzvetkov [15], Kenig and Koenig [13] and Tao [25] have contributed with interesting local and global results for the IVP associated to (3). The best known result till now is due to Tao. He proved local and global well-posedness for data in $H^{1}(\mathbb{R})$.

At this point we should mention that even though the BO equation and the Korteweg-de Vries (KdV) equation,

$$
u_{t}+u_{x x x}+u u_{x}=0
$$

are models for the propagation of waves in the water waves theory, the former one describes the propagation of the wave interphase of two fluids with different densities in deep water meanwhile the second one models the propagation of surface waves on shallow water, the nonlocal character of the dispersion of the BO equation in contrast with the local one of the KdV equation make a huge difference in their analysis. For instance, the methods developed to establish sharp local and global results for the KdV equation (see Kenig, Ponce and Vega [14] and Bourgain [5]) and other nonlinear dispersive equations fail in the $\mathrm{BO}$ equation case.

We will consider the non-homogeneous problem

$$
\left\{\begin{array}{l}
u_{t}+\mathcal{H} u_{x x}=\mathcal{G} h, \quad x \in(0,2 \pi), t>0 \\
u(0, t)=u(2 \pi, t), \quad t>0 \\
u_{x}(0, t)=u_{x}(2 \pi, t), \quad t>0 \\
u(x, 0)=u_{0}(x), \quad x \in(0,2 \pi),
\end{array}\right.
$$

where

$$
\mathcal{G} h(x, t)=g(x)\left(h(x, t)-\int_{0}^{2 \pi} g(y) h(y, t) \mathrm{d} y\right)
$$

for $g(x)$ a smooth function defined in $[0,2 \pi]$, satisfying

$$
2 \pi[g]=\int_{0}^{2 \pi} g(x) \mathrm{d} x=1 .
$$

We can see that the solution of (6) verifies

$$
\int_{0}^{2 \pi} u(x, t) \mathrm{d} x=\int_{0}^{2 \pi} u_{0}(x) \mathrm{d} x, \quad \forall t>0 .
$$

We consider $H_{p}^{s}(0,2 \pi), s \geq 0$, the space of $2 \pi$-periodic functions in $H^{s}(0,2 \pi)$, therefore we have that the Fourier basis

$$
\phi_{k}(x)=\frac{1}{\sqrt{2 \pi}} \mathrm{e}^{i k x}, \quad k \in \mathbb{Z},
$$


is an orthonormal basis of $L^{2}(0,2 \pi)$ and $v \in H_{p}^{s}(0,2 \pi)$ if and only if for

$$
v(x)=\sum_{k \in \mathbb{Z}} v_{k} \phi_{k}(x)
$$

we have that

$$
\sum_{k \in \mathbb{Z}}(1+|k|)^{2 s}\left|v_{k}\right|^{2}<\infty .
$$

Thus we define the norm in $H_{p}^{s}(0,2 \pi)$ as:

$$
\|v\|_{s}=\left(\sum_{k \in \mathbb{Z}}(1+|k|)^{2 s}\left|v_{k}\right|^{2}\right)^{\frac{1}{2}} .
$$

The control problem we will address here is the following: Given $u_{0}, u_{1} \in H_{p}^{s}(0,2 \pi)$ such that $\left[u_{0}\right]=\left[u_{1}\right]$, the problem is to find an external force $\mathcal{G} h \in L^{2}\left(0, T ; H_{p}^{s}(0,2 \pi)\right)$, such that the solution of (6) satisfies $u(x, T)=$ $u_{1}(x)$, for all $x \in(0,2 \pi)$.

In this work we give a positive answer to this problem. More precisely, we show the following result.

Theorem 1.1. Let $T>0$ and $s \geq 0$. Then for each $u_{0}, u_{1} \in H_{p}^{s}(0,2 \pi)$, such that $\left[u_{0}\right]=\left[u_{1}\right]$, there exists a function $h \in L^{2}\left(0, T ; H_{p}^{s}(0,2 \pi)\right)$, such that the solution $u \in C\left([0, T] ; H_{p}^{s}(0,2 \pi)\right)$ of (6) satisfies

$$
u(x, T)=u_{1}(x), \quad x \in(0,2 \pi) .
$$

Moreover, there exists a positive constant $\nu>0$ such that

$$
\|h\|_{L^{2}\left(0, T ; H_{p}^{s}(0,2 \pi)\right)} \leq \nu\left(\left\|u_{0}\right\|_{H_{p}^{s}(0,2 \pi)}+\left\|u_{1}\right\|_{H_{p}^{s}(0,2 \pi)}\right) .
$$

We notice that the controllability results have been intensively studied in the last decade, in particular some results related to the KdV equation have been obtained, we refer to the works of Russell and Zhang [23] and [24], Rosier [22] and the work of Micu [17] for the linearized BBM equation.

To establish our control result we will make use of Ingham's inequality, since the asymptotic gap of the eigenvalues of the Benjamin-Ono equation is infinity. This allows us to obtain a control result for any time $T>0$.

Another interesting problem is the study of the stabilization. In particular, we will study the stabilization of the model one (1) with a feedback law. As in the case of the controllability, the stabilization has been widely studied in the last few years. For instance, for the KdV equation we refer to the works of Russell and Zhang [23] and [24] for the periodic case with a feedback law and the work of Perla, Vasconcellos and Zuazua [20] for the case of a linear localized damping in a bounded domain.

In this work we will study the linearized Benjamin-Ono equation, with a linear feedback and periodic boundary conditions, that is,

$$
\left\{\begin{aligned}
u_{t}+\mathcal{H} u_{x x} & =-\mu \mathcal{G} u, \quad x \in(0,2 \pi), t>0 \\
u(0, t) & =u(2 \pi, t), \quad t>0 \\
u_{x}(0, t) & =u_{x}(2 \pi, t), \quad t>0 \\
u(x, 0) & =u_{0}(x), \quad x \in(0,2 \pi),
\end{aligned}\right.
$$

where $g(x)$ is a nonnegative function in $(0,2 \pi)$ and $\mu>0$.

In this case we can define the energy of the system as

$$
E(t)=\frac{1}{2} \int_{0}^{2 \pi}|u(x, t)|^{2} \mathrm{~d} x .
$$


Multiplying (13) by $u$ and integrating by parts we can easily obtain

$$
\mathrm{E}^{\prime}(t)=-\mu \int_{0}^{2 \pi} u(x, t) \overline{\mathcal{G} u(x, t)} \mathrm{d} x=\int_{0}^{2 \pi} g(x)\left|u(x, t)-\int_{0}^{2 \pi} g(y) u(y, t) \mathrm{d} y\right|^{2} \mathrm{~d} x \leq 0
$$

which implies that the energy is a decreasing function. Thus it is natural to ask whether the associated energy decays exponentially. Here we give an affirmative answer to this question. More precisely.

Theorem 1.2. Let $u_{0} \in L^{2}(0,2 \pi)$, then there exist constants $M, \gamma>0$, independent of $u_{0}$, such that the unique solution $u$ of (13) satisfies

$$
\|u(\cdot, t)-[u]\|_{L^{2}(0,2 \pi)} \leq M \mathrm{e}^{-\gamma t}\left\|u_{0}-\left[u_{0}\right]\right\|_{L^{2}(0,2 \pi)}, \quad \forall t>0 .
$$

We note that both in control and stabilization results, the support of $g$ could be a small interval $(a, b) \subset \subset(0,2 \pi)$. Thus we are acting only a small part of the domain and moreover, since the control and the dissipation has the particular form $\mathcal{G} h$, the solution of (6), (13) and (17) preserves the media of the solution, that is

$$
\int_{0}^{2 \pi} u(x, t) \mathrm{d} x=\int_{0}^{2 \pi} u_{0}(x) \mathrm{d} x, \quad \forall t \in(0, T) .
$$

We shall mention that we have restricted our analysis to the linear problem due to the difficulty to control the nonlinear terms in low regularity periodic Sobolev spaces. More precisely, smoothing effects are not known in this case to overcome the loss of derivatives introduced by the nonlinear term. However, if we do not require to have an exact controllability result in its maximum strength we can obtain the next result whose proof follows the same lines as in [24].

Proposition 1.1. Let $T>0, s \geq 0$ and assuming that

$$
|g(x)|>\beta>0, \quad \forall x \in[0,2 \pi]
$$

then for each $u_{0}, u_{1} \in H_{p}^{s+1}(0,2 \pi)$, such that $\left[u_{0}\right]=\left[u_{1}\right]$, there exists a function $h \in L^{2}\left(0, T ; H_{p}^{s}(0,2 \pi)\right)$, such that the solution $u \in C\left([0, T] ; H_{p}^{s+1}(0,2 \pi)\right)$ of the nonlinear BO problem

$$
\left\{\begin{aligned}
u_{t}+\mathcal{H} u_{x x}+u u_{x} & =\mathcal{G} h, \quad x \in(0,2 \pi), t>0 \\
u(0, t) & =u(2 \pi, t), \quad t>0 \\
u_{x}(0, t) & =u_{x}(2 \pi, t), \quad t>0 \\
u(x, 0) & =u_{0}(x), \quad x \in(0,2 \pi),
\end{aligned}\right.
$$

satisfies

$$
u(x, T)=u_{1}(x), \quad x \in(0,2 \pi) .
$$

We note that the exact controllability for the nonlinear BO equation is a really interesting problem and it may be possible to use the approaches introduced in $[15,25]$ to obtain new results in those spaces.

The two-point initial-boundary value problem for this equation is out of question in its actual form (3) since the nonlocal operator is not well defined in this setting. Still it would be interesting to model this physical situation. Recently, Colliander and Kenig [8] have studied the initial-boundary value problem in the half-line (see also [4]). They have introduced a very promising method to study nonlinear dispersive models in this setting. Again it is not clear whether this problem can be put forward for the BO equation in (3). But it is clear that the physical situation is extremely interesting. In these settings we believe that the problems concerning control and stabilization are more reliable and therefore new models describing the above physical situation will be very helpful.

This note is organized as follows, in Section 2 we will list a series of results needed for the proof of our main result. The results regarding control will be proved in Section 3. Finally, in Section 4 we will prove the stabilization result. 


\section{Some PRELIminary RESUlts}

In this section we will recall some results on the linearized Benjamin-Ono equation. Firstly we recall some basic properties of the Hilbert Transform.

Proposition 2.1. Let $f, g \in L^{2}(-L, L)$. Then we have that

$$
\begin{aligned}
\int_{-L}^{L} f \bar{g} \mathrm{~d} x & =\int_{-L}^{L} \mathcal{H}(f) \overline{\mathcal{H}(g)} \mathrm{d} x, \\
\int_{-L}^{L} f \overline{\mathcal{H}(g)} \mathrm{d} x & =-\int_{-L}^{L} \mathcal{H}(f) \bar{g} \mathrm{~d} x, \\
\mathcal{H}(f \mathcal{H}(g)+\mathcal{H}(f) g) & =\mathcal{H}(f) \mathcal{H}(g)-f g, \\
\mathcal{H}(f)(x) & =i \sum_{k \in \mathbb{Z}} \operatorname{sgn}(k) f_{k} \mathrm{e}^{i x k \pi / L} .
\end{aligned}
$$

where $f_{k}$ is the kth Fourier coefficient of $f$ defined by the formula

$$
f_{k}=\frac{1}{2 L} \int_{-L}^{L} f(x) \mathrm{e}^{-i x k \pi / L} \mathrm{~d} x .
$$

Now, we will study the existence and regularity of the solutions for the linearized Benjamin-Ono equation. In order to study the controllability, we need to study the homogeneous and the non-homogeneous system, thus we have the following.

Proposition 2.2. The operator $A \varphi=-\mathcal{H}\left(\varphi_{x x}\right)$ on $D(A)=H_{p}^{2}$ generates a strongly continuous unitary group $\{S(t)\}_{t \in \mathbb{R}}$ on $L^{2}(0,2 \pi)$.

Proof. Observe that if $\varphi \in D(A)$, then

$$
\varphi(x)=\sum_{n \in \mathbb{Z}} c_{n} \mathrm{e}^{i n x}
$$

therefore

$$
\varphi_{x x}=\sum_{n \in \mathbb{Z}}-n^{2} c_{n} \mathrm{e}^{i n x}
$$

and thus

$$
\mathcal{H}\left(\varphi_{x x}\right)=-\sum_{n \in \mathbb{Z}} i \operatorname{sgn}(n) n^{2} c_{n} \mathrm{e}^{i n x}=(H \varphi)_{x x}
$$

Let $\varphi, \psi \in D(A)$, then

$$
\begin{aligned}
\langle A \varphi, \psi\rangle & =-\int_{0}^{2 \pi} \mathcal{H}\left(\varphi_{x x}\right) \bar{\psi} \mathrm{d} x=\int_{0}^{2 \pi}\left(\varphi_{x x}\right) \overline{\mathcal{H} \psi} \mathrm{d} x \\
& =-\int_{0}^{2 \pi} \varphi_{x} \overline{\mathcal{H}\left(\psi_{x}\right)} \mathrm{d} x+\left.\varphi_{x} \overline{\mathcal{H} \psi}\right|_{x=0} ^{x=2 \pi} \\
& =\int_{0}^{2 \pi} \varphi \overline{\mathcal{H}\left(\psi_{x x}\right)} \mathrm{d} x-\left.\varphi \overline{\mathcal{H}\left(\psi_{x}\right)}\right|_{x=0} ^{x=2 \pi} \\
& =\left\langle\varphi, \mathcal{H} \psi_{x x}\right\rangle=-\langle\varphi, A \psi\rangle,
\end{aligned}
$$

this implies that $A$ is skew-adjoint. Moreover, since

$$
\langle A \varphi, \varphi\rangle=-\langle\varphi, A \varphi\rangle
$$


we have that

$$
\langle A \varphi, \varphi\rangle=0 .
$$

Therefore $A$ generates a unitary group of isometries $\{S(t)\}_{t \in \mathbb{R}}$ (see [7], Th. 3.2.3), and the proof is completed.

Thus, from classical results of semigroup theory, we have the following result for the homogeneous and non-homogeneous equation.

We first consider the homogeneous case,

$$
\left\{\begin{array}{l}
\varphi_{t}+\mathcal{H} \varphi_{x x}=0, \quad x \in(0,2 \pi), t>0 \\
\varphi(0, t)=\varphi(2 \pi, t), \quad t>0 \\
\varphi_{x}(0, t)=\varphi_{x}(2 \pi, t), \quad t>0 \\
\varphi(x, 0)=\varphi_{0}(x), \quad x \in(0,2 \pi)
\end{array}\right.
$$

For this problem we have.

Lemma 2.1. Let $\varphi_{0} \in L^{2}(0,2 \pi)$. Then there exists a unique solution $\varphi \in C\left(\mathbb{R}, L^{2}(0,2 \pi)\right)$ of (23). If $\varphi_{0} \in$ $H_{p}^{2}(0,2 \pi)$, then $\varphi \in C^{1}\left(\mathbb{R}, L^{2}(0,2 \pi)\right) \cap C\left(\mathbb{R}, H_{p}^{2}(0,2 \pi)\right)$.

Moreover, if $\varphi \in H_{p}^{s}(0,2 \pi)$, then there exists a unique solution of (23) such that

$$
\varphi \in C\left(\mathbb{R}, H_{p}^{s}(0,2 \pi)\right)
$$

For the non-homogeneous problem we have.

Lemma 2.2. Let $\varphi_{0} \in H_{p}^{s}(0,2 \pi)$ and $h \in L^{2}\left(0, T ; H_{p}^{s}(0,2 \pi)\right)$, with $s \geq 0$. Then there exists a unique solution $\varphi \in C\left([0, T], H_{p}^{s}(0,2 \pi)\right)$ of $(6)$.

The proof of the above results is obtained from classical results on the semigroup theory, we refer to the books by Cazenave and Haraux [7] or Pazy [19] for details.

Remark 2.1. Let $h \in L^{2}\left(0, T ; H_{p}^{s}(0,2 \pi)\right)$, then if $g$ is a smooth function, we have that $\mathcal{G} h \in L^{2}\left(0, T ; H_{p}^{s}(0,2 \pi)\right)$. In fact,

$$
\begin{aligned}
\|\mathcal{G} h\|_{L^{2}\left(0, T ; H_{p}^{s}(0,2 \pi)\right)} & =\int_{0}^{T}\|\mathcal{G} h(\cdot, t)\|_{H_{p}^{s}(0,2 \pi)}^{2} \mathrm{~d} t \\
& =\int_{0}^{T}\left\|g(\cdot)\left(h(\cdot, t)-\int_{0}^{2 \pi} g(y) h(y, t) \mathrm{d} y\right)\right\|_{H_{p}^{s}(0,2 \pi)}^{2} \mathrm{~d} t<\infty .
\end{aligned}
$$

To study the stabilization with a feedback law, we consider the system

$$
\left\{\begin{array}{rlrl}
u_{t}+\mathcal{H} u_{x x} & =-\mu \mathcal{G} u, & & (x, t) \in(0,2 \pi) \times(0, \infty) \\
u(0, t) & =u(2 \pi, t), & & t>0 \\
u_{x}(0, t) & =u_{x}(2 \pi, t), & t>0 \\
u(x, 0) & =u_{0}(x), & & x \in(0,2 \pi),
\end{array}\right.
$$

for a positive constant $\mu$ and $g$ a nonnegative smooth function. In particular we are interested in the case where the support of $g$ is contained in a small interval of $(0,2 \pi)$.

Thus, we have the following result of existence and regularity for this model.

Lemma 2.3. Let $u_{0} \in H_{p}^{2}(0,2 \pi)$, then the problem $(24)$ has a unique solution $u \in C\left((0, \infty) ; H_{p}^{2}(0,2 \pi)\right) \cap$ $C^{1}\left((0, \infty) ; L^{2}(0,2 \pi)\right)$. Moreover, if $u_{0} \in L^{2}(0,2 \pi)$, we have that $u \in C\left((0, \infty) ; L^{2}(0,2 \pi)\right)$. 
Proof. We can note that the operator $A=-\mathcal{H} u_{x x}$ generates a strongly continuous semigroup, and since the map $h \rightarrow \mathcal{G} h$ is a bounded perturbation of $A$, we have that the operator $A-\mu \mathcal{G} h$ generates a strongly continuous semigroup.

Our main tool is the so-called Ingham's inequality, we recall this result (see [10]).

Theorem 2.1. Let $\left\{\lambda_{n}\right\}_{n=-\infty}^{\infty}$ be a strictly increasing sequence of real numbers and I be a bounded interval. Consider the sums of the form

$$
f(t)=\sum_{n=-\infty}^{\infty} b_{n} \mathrm{e}^{i \lambda_{n} t}, \quad t \in I .
$$

Assume that there exists $\gamma_{1}>0$ such that the "gap condition"

$$
\lambda_{n+1}-\lambda_{n} \geq \gamma_{1}, \quad \forall n \in \mathbb{Z},
$$

holds, then there exist constants $c_{1}, c_{2}>0$, such that for every bounded interval I of length $|I|>2 \pi / \gamma_{1}$

$$
c_{1} \sum_{n=-\infty}^{\infty}\left|b_{n}\right|^{2} \leq \int_{I}|f(t)|^{2} \mathrm{~d} t \leq c_{2} \sum_{n=-\infty}^{\infty}\left|b_{n}\right|^{2} .
$$

Remark 2.2. In the above theorem we can replace the gap condition by a more general condition called "asymptotic gap condition", that is,

$$
\limsup _{n \rightarrow \infty}\left|\lambda_{n+1}-\lambda_{n}\right| \geq \gamma_{1}
$$

We will also recall some useful results on Riesz basis. In what follows $H$ is a Hilbert space.

Definition 2.1. A sequence $\left\{x_{n}\right\}_{n} \subset H$ is called complete if given $x \in H$ such that

$$
\left(x, x_{n}\right)=0, \quad \forall n,
$$

then $x \equiv 0$.

Remark 2.3. The following statements are equivalent:

(1) $\left\{x_{n}\right\}_{n}$ is complete in $H$;

(2) $\operatorname{span}\left\{x_{n}: n\right\}$ is dense in $H$.

Definition 2.2. We say that the basis $\left\{x_{n}\right\}_{n}$ and $\left\{y_{n}\right\}_{n}$ are equivalent if and only if we have that the series $\sum_{n} c_{n} x_{n}$ converges if and only if the series $\sum_{n} c_{n} y_{n}$ converges.

Definition 2.3. A basis $\left\{x_{n}\right\}_{n}$ of $H$ is a Riesz basis if and only if it is equivalent to an orthonormal basis of $H$.

Theorem 2.2. The following statements are equivalent:

(1) $\left\{x_{n}\right\}_{n}$ is a Riesz basis.

(2) $\left\{x_{n}\right\}_{n}$ is complete and there exists an inner product in $H$, denoted by $(\cdot, \cdot)$, equivalent to the inner product of $H$ such that

$$
\left(x_{i}, x_{j}\right)=\delta_{i j}
$$

(3) $\left\{x_{n}\right\}_{n}$ is complete and there exist two positive constants $A$ and $B$, such that

$$
A \sum_{n}\left|c_{n}\right|^{2} \leq\left|\sum_{k=1}^{n} c_{k} x_{k}\right|^{2} \leq B \sum_{n}\left|c_{n}\right|^{2}
$$

for all $n \in \mathbb{N}$ and any $c_{1}, \ldots, c_{n}$ scalars. 
Definition 2.4. We say that the sequence $\left\{x_{n}\right\}_{n}$ is minimal in $H$ if each element of the sequence lies outside the closed linear span of the others.

Definition 2.5. We say that the sequences $\left\{x_{n}\right\}_{n}$ and $\left\{y_{n}\right\}_{n}$ are biorthogonal if

$$
\left(x_{i}, y_{j}\right)=\delta_{i j}, \quad \forall i, j .
$$

Now we have the following results.

Proposition 2.3. Let $\left\{x_{n}\right\}_{n}$ be a sequence in $H$. Then $\left\{x_{n}\right\}_{n}$ is biorthogonal iff it is minimal.

Proposition 2.4. Let $\left\{x_{n}\right\}_{n}$ be a minimal sequence in $H$. Then $\left\{x_{n}\right\}_{n}$ is complete in $H$ iff the biorthogonal sequence is uniquely determined.

\section{Proof of the CONTRol Results}

To prove our control results, we will need to establish some technical results useful in the proof. We first notice that, without loss of generality one can consider the case $u_{0}=0$. In fact, let $u_{0}, u_{1} \in H_{p}^{2}(0,2 \pi)$, such that $\left[u_{0}\right]=\left[u_{1}\right]$, then if $f=\mathcal{G} h$ is the control which leads the solution from initial data zero to the final state $u_{1}-S(T) u_{0}$, where $S(t)$ is the group generated by the differential operator corresponding to the linearized BO equation, then we have that this control leads the solution from $u_{0}$ to the final state $u_{1}$.

Lemma 3.1. Let $u_{1} \in H_{p}^{s}(0,2 \pi)$ with $\left[u_{1}\right]=0$. Then, there exists $h \in L^{2}\left(0, T ; H_{p}^{s}(0,2 \pi)\right)$, such that the solution of (6), with initial data $u_{0}=0$, satisfies $u(T)=u_{1}$ if and only if

$$
\int_{0}^{T}\langle\mathcal{G} h(\cdot, t), \bar{\varphi}(\cdot, t)\rangle_{H_{p}^{s} \times\left(H_{p}^{s}\right)^{\prime}} \mathrm{d} t-\left\langle u_{1}, \bar{\varphi}_{0}\right\rangle_{H_{p}^{s} \times\left(H_{p}^{s}\right)^{\prime}}=0
$$

for any $\varphi_{0} \in\left(H_{p}^{s}(0,2 \pi)\right)^{\prime}$, the dual space of $H_{p}^{s}(0,2 \pi)$, where $\varphi$ is the solution of the adjoint system with data $\varphi_{0}$,

$$
\left\{\begin{array}{l}
\varphi_{t}+\mathcal{H} \varphi_{x x}=0, \quad x \in(0,2 \pi), t>0 \\
\varphi(0, t)=\varphi(2 \pi, t), \quad t>0 \\
\varphi_{x}(0, t)=\varphi_{x}(2 \pi, t), \quad t>0 \\
\varphi(x, T)=\varphi_{0}(x), \quad x \in(0,2 \pi) .
\end{array}\right.
$$

Proof. Let $\varphi_{0}$ and $h$ be smooth functions and $\varphi$ be the solution of the adjoint system (30) with final data $\varphi_{0}$. Then, multiplying (6) by $\varphi$ and integrating, we obtain

$$
\begin{aligned}
\int_{0}^{T} \int_{0}^{2 \pi} \mathcal{G} h \bar{\varphi} \mathrm{d} x \mathrm{~d} t & =\int_{0}^{T} \int_{0}^{2 \pi}\left(u_{t}+\mathcal{H} u_{x x}\right) \bar{\varphi} \mathrm{d} x \mathrm{~d} t \\
& =-\int_{0}^{T} \int_{0}^{2 \pi} u \bar{\varphi}_{t} \mathrm{~d} x \mathrm{~d} t+\left.\int_{0}^{2 \pi} u \bar{\varphi}\right|_{t=0} ^{t=T} \mathrm{~d} x-\int_{0}^{T} \int_{0}^{2 \pi} u_{x x} \overline{\mathcal{H} \varphi} \mathrm{d} x \mathrm{~d} t \\
& =\int_{0}^{2 \pi} u(T) \bar{\varphi}_{0} \mathrm{~d} x-\int_{0}^{2 \pi} u_{0} \bar{\varphi}(0) \mathrm{d} x=\int_{0}^{2 \pi} u(T) \bar{\varphi}_{0} \mathrm{~d} x
\end{aligned}
$$

that is

$$
\int_{0}^{T} \int_{0}^{2 \pi} \mathcal{G} h \bar{\varphi} \mathrm{d} x \mathrm{~d} t=\int_{0}^{2 \pi} u(T) \bar{\varphi}_{0} \mathrm{~d} x
$$

for all smooth data. Therefore by using density arguments we conclude the proof.

It is well known that the existence of biorthogonal basis implies the existence of a control. Next result describes this relationship. 
Lemma 3.2. Let

$$
u_{1}(x)=\sum_{m \in \mathbb{Z}} c_{m} \phi_{m}(x) \in H_{p}^{s}(0,2 \pi),
$$

be a function such that $\left[u_{1}\right]=0$ (that is $c_{0}=0$ ), then we have that (6) is exactly controllable to $u_{1}$, that is, $u(x, T)=u_{1}(x), x \in(0,2 \pi)$, if and only if there exists $h \in L^{2}\left(0, T ; H_{p}^{s}(0,2 \pi)\right)$ such that

$$
\int_{0}^{T} \int_{0}^{2 \pi} \mathcal{G} h(x, t) \bar{\phi}_{n}(x) \mathrm{e}^{i s g n(n) n^{2}(T-t)} \mathrm{d} x \mathrm{~d} t=c_{n}, \quad \forall n \in \mathbb{Z} .
$$

Proof. It is enough to consider $\varphi_{0}(x)=\phi_{n}(x)$. Since $\varphi(x, T)=\phi_{n}(x) \mathrm{e}^{-i \lambda_{n}(T-t)}$, where $\lambda_{n}=i \operatorname{sgn}(n) n^{2}$, the identity (29) in Lemma 3.1 implies that

$$
\int_{0}^{T} \int_{0}^{2 \pi} \mathcal{G} h(x, t) \bar{\phi}_{n}(x) \mathrm{e}^{i \lambda_{n}(T-t)} \mathrm{d} x \mathrm{~d} t=\int_{0}^{2 \pi} \bar{\phi}_{n}(x)\left(\sum_{m \in \mathbb{Z}} c_{m} \phi_{m}(x)\right) \mathrm{d} x=c_{n} .
$$

Next we will prove the control result.

Proof of Theorem 1.1. First, we will show that $\left\{\mathrm{e}^{i \lambda_{n} t}\right\}_{n \in \mathbb{Z}}$ is a Riesz basis for its closed span $\mathcal{P}$ in $L^{2}(0, T)$. In fact, due to the asymptotic gap of the family of eigenvalues, that is

$$
\lim _{|n| \rightarrow \infty}\left|\lambda_{n+1}-\lambda_{n}\right|=\left|\operatorname{sgn}(n+1)(n+1)^{2}-\operatorname{sgn}(n) n^{2}\right|=\infty,
$$

from the Ingham's inequality we have that the family is complete in $\mathcal{P}$ and there exist positive constants $A, B$ such that

$$
A \sum_{n \in \mathbb{Z}}\left|c_{n}\right|^{2} \leq \int_{I}\left|\sum_{n \in \mathbb{Z}} c_{n} \mathrm{e}^{-i \lambda_{n} t}\right|^{2} \mathrm{~d} t \leq B \sum_{n \in \mathbb{Z}}\left|c_{n}\right|^{2}
$$

and therefore $\left\{\mathrm{e}^{i \lambda_{n} t}\right\}_{n \in \mathbb{Z}}$ is a Riesz basis of $\mathcal{P}$.

Therefore we have the existence of a biorthogonal basis due to the minimality of the set. Thus, there exists a dual basis $\left\{q_{m}\right\}_{m}$ (which is unique), such that

$$
\int_{0}^{T} q_{m} \mathrm{e}^{i \lambda_{k} t} \mathrm{~d} t=\delta_{m k}
$$

Hence, if we consider the function

$$
h=\sum_{n} h_{n} q_{n} \mathcal{G} \phi_{n}
$$

for suitable $h_{n}$, it will follow that

$$
\begin{aligned}
\int_{0}^{T} \int_{0}^{2 \pi} \mathcal{G} h \bar{\phi}_{k} \mathrm{e}^{i \lambda_{k}(T-t)} \mathrm{d} x \mathrm{~d} t & =\sum_{n} h_{n} \int_{0}^{T} \mathrm{e}^{i \lambda_{k}(T-t)} q_{n} \int_{0}^{2 \pi} \bar{\phi}_{k} \mathcal{G}\left(\mathcal{G} \phi_{n}\right) \mathrm{d} x \mathrm{~d} t \\
& =h_{k} \int_{0}^{2 \pi} \bar{\phi}_{k} \mathcal{G}\left(\mathcal{G} \phi_{k}\right) \mathrm{d} x \\
& =h_{k} \int_{0}^{2 \pi}\left|\mathcal{G} \phi_{k}\right|^{2} \mathrm{~d} x \\
& =h_{k}\left\|\mathcal{G} \phi_{k}\right\|_{L^{2}(0,2 \pi)}^{2} .
\end{aligned}
$$


We will show that

$$
\left\|\mathcal{G} \phi_{k}\right\|_{L^{2}(0,2 \pi)}^{2}>\alpha>0, \forall k \neq 0
$$

Assuming this, we deduce from Lemma 3.2 applied to

$$
u_{1}(x)=\sum_{n \in \mathbb{Z}} c_{n} \phi_{n}(x) \in H_{p}^{s}(0,2 \pi)
$$

that it is enough to consider $h_{k}$ satisfying

$$
c_{k}=h_{k}\left\|\mathcal{G} \phi_{k}\right\|_{L^{2}(0,2 \pi)}^{2}, \forall k \neq 0 .
$$

Next we prove $h \in L^{2}\left(0, T ; H_{p}^{s}(0,2 \pi)\right)$ and inequality (37).

It is easy to see that

$$
\beta_{n}=\left\|\mathcal{G} \phi_{n}\right\|_{L^{2}(0,2 \pi)}^{2}=\int_{0}^{2 \pi}\left|g(x)\left(\mathrm{e}^{i n x}-\int_{0}^{2 \pi} g(y) \mathrm{e}^{i n y} \mathrm{~d} y\right)\right|^{2} \mathrm{~d} x>0, \quad \forall n \in \mathbb{Z}^{*} .
$$

Since

$$
\lim _{|n| \rightarrow \infty} \beta_{n}=\int_{0}^{2 \pi}|g(x)|^{2} \mathrm{~d} x \neq 0
$$

we deduce that there exists a positive constant $\beta$ such that

$$
\beta_{n}>\beta>0, \quad \forall n \in \mathbb{Z}^{*} .
$$

Next we show that the function $h$ defined in $(36)$ and $(38)$ is in $L^{2}\left(0, T ; H_{p}^{s}(0,2 \pi)\right)$. We proceed as in [24]. Assuming

$$
\mathcal{G} \phi_{n}=\sum_{m \in \mathbb{Z}} a_{m n} \phi_{m}
$$

the definition (36) implies that

$$
h=\sum_{n \in \mathbb{Z}} \sum_{m \in \mathbb{Z}} h_{n} q_{n} a_{m n} \phi_{m}=\sum_{n \in \mathbb{Z}} \sum_{m \in \mathbb{Z}} \frac{c_{n}}{\beta_{n}} q_{n} a_{m n} \phi_{m}
$$

Then

$$
\begin{aligned}
\|h\|_{L^{2}\left(0, T ; H_{p}^{s}(0,2 \pi)\right)}^{2} & =\int_{0}^{T}\left\|\sum_{n \in \mathbb{Z}} \sum_{m \in \mathbb{Z}} \frac{c_{n}}{\beta_{n}} q_{n}(t) a_{m n} \phi_{m}\right\|_{H_{p}^{s}(0,2 \pi)}^{2} \mathrm{~d} t \\
& =\int_{0}^{T} \sum_{m \in \mathbb{Z}}(1+|m|)^{2 s}\left|\sum_{n \in \mathbb{Z}} q_{n}(t) \frac{c_{n}}{\beta_{n}} a_{m n}\right|^{2} \mathrm{~d} t \\
& \leq c \sum_{m \in \mathbb{Z}}(1+|m|)^{2 s} \sum_{n \in \mathbb{Z}}\left|\frac{c_{n}}{\beta_{n}} a_{m n}\right|^{2} \\
& \leq c \sum_{n \in \mathbb{Z}}\left|\frac{c_{n}}{\beta_{n}}\right|^{2} \sum_{m \in \mathbb{Z}}(1+|m|)^{2 s}\left|a_{m n}\right|^{2} .
\end{aligned}
$$

Setting

$$
g(x)=\sum_{k \in \mathbb{Z}} g_{k} \phi_{k}(x)
$$


it follows that

$$
\begin{aligned}
\left|a_{m n}\right| & =\left|\left(\mathcal{G} \phi_{n}, \phi_{m}\right)_{L^{2}(0,2 \pi)}\right| \\
& =\left|\left(g \phi_{n}, \phi_{m}\right)_{L^{2}(0,2 \pi)}-\left(\phi_{n}, g\right)_{L^{2}(0,2 \pi)}\left(\phi_{m}, g\right)_{L^{2}(0,2 \pi)}\right| \\
& =\left|\sum_{k \in \mathbb{Z}} g_{k}\left(\phi_{k} \phi_{n}, \phi_{m}\right)_{L^{2}(0,2 \pi)}-g_{n} g_{m}\right| \\
& =\left|\frac{1}{2 \pi} g_{k}\left(\phi_{k} \phi_{n}, \phi_{m}\right)_{L^{2}(0,2 \pi)}-g_{n} g_{m}\right| \\
& =\left|\frac{1}{2 \pi} g_{m-n}-g_{n} g_{m}\right| \\
& \leq \frac{1}{2 \pi}\left|g_{m-n}\right|+\left|g_{n}\right|\left|g_{m}\right| .
\end{aligned}
$$

Therefore, there exists a positive constant $c_{1}$ such that

$$
\begin{aligned}
\sum_{m \in \mathbb{Z}}(1+|m|)^{2 s}\left|a_{m n}\right|^{2} & \leq c_{1} \sum_{m \in \mathbb{Z}}(1+|m|)^{2 s}\left(\left|g_{m-n}\right|^{2}+\left|g_{n}\right|^{2}\left|g_{m}\right|^{2}\right) \\
& \leq c_{1} \sum_{m \in \mathbb{Z}}(1+|m|)^{2 s}\left|g_{m-n}\right|^{2}+c_{1}\left|g_{n}\right|^{2} \sum_{m \in \mathbb{Z}}(1+|m|)^{2 s}\left|g_{m}\right|^{2} \\
& \leq c_{2}(1+|n|)^{2 s} \sum_{k \in \mathbb{Z}}(1+|k|)^{2 s}\left|g_{k}\right|^{2}+c_{1}\left|g_{n}\right|^{2}\|g\|_{s}^{2} \\
& \leq c_{3}\left((1+|n|)^{2 s}+\left|g_{n}\right|^{2}\right)\|g\|_{s}^{2}
\end{aligned}
$$

Thus, from (43) and (45) we obtain that

$$
\begin{aligned}
\|h\|_{L^{2}\left(0, T ; H_{p}^{s}(0,2 \pi)\right)}^{2} & \leq c \sum_{n \in \mathbb{Z}}\left|\frac{c_{n}}{\beta_{n}}\right|^{2} \sum_{m \in \mathbb{Z}}(1+|m|)^{2 s}\left|a_{m n}\right|^{2} \\
& \leq c c_{3} \sum_{n \in \mathbb{Z}}\left|\frac{c_{n}}{\beta_{n}}\right|^{2}\left((1+|n|)^{2 s}+\left|g_{n}\right|^{2}\right)\|g\|_{s}^{2} \\
& \leq \frac{c c_{3}}{\beta^{2}}\|g\|_{s}^{2} \sum_{n \in \mathbb{Z}}\left|c_{n}\right|^{2}\left((1+|n|)^{2 s}+\left|g_{n}\right|^{2}\right) \\
& \leq \frac{c c_{3}}{\beta^{2}}\|g\|_{s}^{2}\left(\left\|u_{1}\right\|_{s}^{2}+\sum_{n \in \mathbb{Z}}\left|c_{n}\right|^{2}\left|g_{n}\right|^{2}\right) \\
& \leq\|g\|_{s}^{2}\left(\frac{c c_{3}}{\beta^{2}}+\max _{n \in \mathbb{Z}}\left|g_{n}\right|^{2}\right)\left\|u_{1}\right\|_{s}^{2} .
\end{aligned}
$$

Therefore, considering

we conclude the proof.

$$
\nu=\|g\|_{s}^{2}\left(\frac{c c_{3}}{\beta^{2}}+\max _{n \in \mathbb{Z}}\left|g_{n}\right|^{2}\right),
$$

Now, we will give an sketch of the proof of Proposition 1.1.

Proof of Proposition 1.1. In what follows we will give a sketch of the proof of Proposition 1.1, which is similar to the one given in [24] (Th. 1.1). 
Let $u_{0}, u_{1} \in H_{p}^{s+1}(0,2 \pi)$, verifying $\left[u_{0}\right]=\left[u_{1}\right]$, then from the controllability result, there exists $h_{1} \in$ $L^{2}\left(0, T ; H_{p}^{s+1}(0,2 \pi)\right)$, such that the solution of $(6)$ verifies

$$
u(T)=u_{1} .
$$

Moreover, we can see that $u \in C\left([0, T] ; H_{p}^{s+1}(0,2 \pi)\right)$, therefore we have that the nonlinear term $u u_{x}$ verifies that

$$
u u_{x} \in L^{2}\left(0, T ; H_{p}^{s}(0,2 \pi)\right)
$$

then, adding this term to the equation we have that $u$ verifies $(47)$ and

$$
\left\{\begin{array}{l}
u_{t}+\mathcal{H} u_{x x}+u u_{x}=\mathcal{G} h_{1}+u u_{x}, \quad x \in(0,2 \pi), t>0 \\
u(0, t)=u(2 \pi, t), \quad t>0 \\
u_{x}(0, t)=u_{x}(2 \pi, t), \quad t>0 \\
u(x, 0)=u_{0}(x), \quad x \in(0,2 \pi) .
\end{array}\right.
$$

Thus, it is enough to prove that there exists $h_{2} \in L^{2}\left(0, T ; H_{p}^{s}(0,2 \pi)\right)$ such that

$$
u u_{x}=G h_{2}
$$

or equivalently

$$
u(x, t) u_{x}(x, t)=g(x)\left(h_{2}(x, t)-\int_{0}^{2 \pi} h_{2}(y, t) g(y, t) \mathrm{d} y\right),
$$

and since $|g(x)| \geq \beta>0$ in $(0,2 \pi)$, we can write (50) as

$$
\frac{1}{g(x)} u(x, t) u_{x}(x, t)=h_{2}(x, t)-\int_{0}^{2 \pi} h_{2}(y, t) g(y, t) \mathrm{d} y .
$$

Now, we will study the existence of $h_{2}$. We define the space $Y_{g}^{s}$ as the space $H_{p}^{s}(0,2 \pi)$ endowed with the norm

$$
\|v\|_{Y_{g}^{s}}=(g v, v)_{H_{p}^{s}}^{\frac{1}{2}} .
$$

For each $v \in Y_{g}^{s}$ we define the map

$$
\widetilde{G}: Y_{g}^{s} \rightarrow Y_{g}^{s}, \quad \widetilde{G} v(x)=v(x)-\int_{0}^{2 \pi} v(y) g(y) \mathrm{d} y .
$$

Then, as in [24], it is easy to prove that

(1) $\widetilde{G}$ is a Fredholm operator.

(2) $\widetilde{G}^{*}=\widetilde{G}$.

(3) $N(\widetilde{G})=\langle\{1\}\rangle$.

(4) $\widetilde{G}$ has a bounded inverse considered as an operator from $Y_{g}^{s} \backslash N(\widetilde{G})$ to $\widetilde{G}$.

Then, since for every $t \in(0, T)$

$$
\left(\frac{u u_{x}}{g}, 1\right)_{Y_{g}^{s}}=\int_{0}^{2 \pi} u(y, t) u_{x}(y, t) \mathrm{d} y=0
$$


we have that

$$
\frac{u u_{x}}{g} \in N(\widetilde{G})^{\perp}=N\left(\widetilde{G}^{*}\right)^{\perp}=R(\widetilde{G}),
$$

then for each $t \in(0, T)$ there exists a function $h_{2}(\cdot, t)$ verifying

$$
\frac{u(\cdot, t) u_{x}(\cdot, t)}{g(\cdot)}=\widetilde{G} h_{2}(\cdot, t)
$$

and since the inverse of $\widetilde{G}$ has a bounded inverse, we obtain that

$$
h_{2} \in L^{2}\left(0, T ; H_{p}^{s}(0,2 \pi)\right) \text {, }
$$

thus, considering the control function $h=h_{1}+h_{2}$, we complete the proof.

\section{Proof of the stabilization Result}

Now we will prove Theorem 1.2 regarding stabilization.

Let $T>0$ and $u_{0} \in L^{2}(0,2 \pi)$ such that $\left[u_{0}\right]=0$. Then we consider the problems

$$
\left\{\begin{aligned}
w_{t}+\mathcal{H} w_{x x} & =\mathcal{G} h, \quad x \in(0,2 \pi), t>0 \\
w(0, t) & =w(2 \pi, t), \quad t>0 \\
w_{x}(0, t) & =w_{x}(2 \pi, t), \quad t>0 \\
w(x, 0) & =0, \quad x \in(0,2 \pi)
\end{aligned}\right.
$$

such that

$$
w(x, T)=u(x, T), \quad x \in(0,2 \pi),
$$

and

We will assume that

$$
\left\{\begin{aligned}
u_{t}+\mathcal{H} u_{x x} & =-\mu \mathcal{G} u, \quad x \in(0,2 \pi), t>0 \\
u(0, t) & =u(2 \pi, t), \quad t>0 \\
u_{x}(0, t) & =u_{x}(2 \pi, t), \quad t>0 \\
u(x, 0) & =u_{0}(x), \quad x \in(0,2 \pi) .
\end{aligned}\right.
$$

then we have that the solutions

$$
u_{0} \in H_{p}^{2}(0,2 \pi)
$$

$$
u, w \in C\left([0, T] ; H_{p}^{2}(0,2 \pi)\right) \cap C^{1}\left(0, T ; L^{2}(0,2 \pi)\right)
$$

and

$$
h \in L^{2}\left(0, T ; H_{p}^{2}(0,2 \pi)\right) .
$$

Then, multiplying the first equation in (54) by $u$ and integrating with respect to $x$ it follows that

$$
\frac{1}{2} \frac{\mathrm{d}}{\mathrm{d} t}\left(\int_{0}^{2 \pi}|u(x, t)|^{2} \mathrm{~d} x\right)=-\mu \int_{0}^{2 \pi} \mathcal{G} u(x, t) \bar{u}(x, t) \mathrm{d} x,
$$

that is,

$$
\frac{1}{2} \int_{0}^{2 \pi}|u(x, T)|^{2} \mathrm{~d} x-\frac{1}{2} \int_{0}^{2 \pi}\left|u_{0}(x)\right|^{2} \mathrm{~d} x=-\mu \int_{0}^{T} \int_{0}^{2 \pi} \mathcal{G} u(x, t) \bar{u}(x, t) \mathrm{d} x \mathrm{~d} t .
$$


On the other hand,

$$
\begin{aligned}
\int_{0}^{T} \int_{0}^{2 \pi} \mathcal{G} h \bar{u} \mathrm{~d} x \mathrm{~d} t & =\int_{0}^{T} \int_{0}^{2 \pi}\left(w_{t}+\mathcal{H} w_{x x}\right) \bar{u} \mathrm{~d} x \mathrm{~d} t \\
& =-\int_{0}^{T} \int_{0}^{2 \pi} w \overline{u_{t}} \mathrm{~d} x \mathrm{~d} t+\left.\int_{0}^{2 \pi} w \bar{u}\right|_{t=0} ^{t=T} \mathrm{~d} x+\int_{0}^{T} \int_{0}^{2 \pi} \mathcal{H} w_{x x} \bar{u} \mathrm{~d} x \mathrm{~d} t \\
& =\int_{0}^{T} \int_{0}^{2 \pi} w \overline{\left(\mathcal{H} u_{x x}+\mu \mathcal{G} u\right)} \mathrm{d} x \mathrm{~d} t+\int_{0}^{2 \pi}|u(T)|^{2} \mathrm{~d} x \mathrm{~d} t+\int_{0}^{T} \int_{0}^{2 \pi} \mathcal{H} w_{x x} \bar{u} \mathrm{~d} x \mathrm{~d} t \\
& =\int_{0}^{T} \int_{0}^{2 \pi} w \overline{\mu \mathcal{G} u} \mathrm{~d} x \mathrm{~d} t+\int_{0}^{2 \pi}|u(T)|^{2} \mathrm{~d} x .
\end{aligned}
$$

Thus

$$
\int_{0}^{2 \pi}|u(T)|^{2} \mathrm{~d} x=\int_{0}^{T} \int_{0}^{2 \pi}(h-\mu w) \overline{\mathcal{G} u} \mathrm{~d} x \mathrm{~d} t \leq\|h-\mu w\|_{L^{2}((0, T) \times(0,2 \pi))}\|\mathcal{G} u\|_{L^{2}((0, T) \times(0,2 \pi))} .
$$

We also have that

and

$$
\|h\|_{L^{2}\left(0, T ; L^{2}(0,2 \pi)\right)} \leq \nu\|u(\cdot, T)\|_{L^{2}(0,2 \pi)},
$$

$$
\|w\|_{L^{2}\left(0, T ; L^{2}(0,2 \pi)\right)} \leq \mu\|u(\cdot, T)\|_{L^{2}(0,2 \pi)} .
$$

Using (59) and (60) we deduce easily that there exists a positive constant $C>0$, such that,

$$
\|h-\mu u\|_{L^{2}\left(0, T ; L^{2}(0,2 \pi)\right)} \leq C\|u(\cdot, T)\|_{L^{2}(0,2 \pi)} .
$$

Now, from (58) and (61) we have that

$$
\|u(\cdot, T)\|_{L^{2}(0,2 \pi)} \leq C\|\mathcal{G} u\|_{L^{2}((0, T) \times(0,2 \pi))} .
$$

Note that

$$
\begin{aligned}
\|\mathcal{G} u\|_{L^{2}((0, T) \times(0,2 \pi))}^{2} & =\int_{0}^{T} \int_{0}^{2 \pi}\left|g(x)\left(u(x, t)-\int_{0}^{2 \pi} g(y) u(y, t) \mathrm{d} y\right)\right|^{2} \mathrm{~d} x \mathrm{~d} t \\
& =\int_{0}^{T} \int_{0}^{2 \pi} g(x)^{2}\left|\left(u(x, t)-\int_{0}^{2 \pi} g(y) u(y, t) \mathrm{d} y\right)\right|^{2} \mathrm{~d} x \mathrm{~d} t \\
& \leq\|g\|_{\infty} \int_{0}^{T} \int_{0}^{2 \pi} g(x)\left|\left(u(x, t)-\int_{0}^{2 \pi} g(y) u(y, t) \mathrm{d} y\right)\right|^{2} \mathrm{~d} x \mathrm{~d} t \\
& =\|g\|_{\infty} \int_{0}^{T} \int_{0}^{2 \pi} \mathcal{G} u(x, t) \bar{u}(x, t) \mathrm{d} x \mathrm{~d} t .
\end{aligned}
$$

Combining (58) and (63) yields

$$
\|u(\cdot, T)\|_{L^{2}(0,2 \pi)}^{2} \leq C\|g\|_{\infty} \int_{0}^{T} \int_{0}^{2 \pi} \mathcal{G} u(x, t) \bar{u}(x, t) \mathrm{d} x \mathrm{~d} t .
$$

Thus, using(56) and (63) we obtain that

$$
\|u(\cdot, T)\|_{L^{2}(0,2 \pi)}^{2}-\left\|u_{0}\right\|_{L^{2}(0,2 \pi)}^{2} \leq-\mu\left(C\|g\|_{\infty}\right)^{-1}\|u(\cdot, T)\|_{L^{2}(0,2 \pi)}^{2},
$$


that is,

or equivalently

$$
\left(1+\mu\left(C\|g\|_{\infty}\right)^{-1}\right)\|u(\cdot, T)\|_{L^{2}(0,2 \pi)}^{2} \leq\left\|u_{0}\right\|_{L^{2}(0,2 \pi)}^{2},
$$

$$
\|u(\cdot, T)\|_{L^{2}(0,2 \pi)}^{2} \leq \frac{C\|g\|_{\infty}}{C\|g\|_{\infty}+\mu}\left\|u_{0}\right\|_{L^{2}(0,2 \pi)}^{2} .
$$

Since the last inequality is valid for every time $T>0$, we conclude that there exist constants $M, \gamma>0$ such that

$$
\|u(\cdot, t)\|_{L^{2}(0,2 \pi)} \leq M \mathrm{e}^{-\gamma t}\left\|u_{0}\right\|_{L^{2}(0,2 \pi)}, \quad \forall t>0 .
$$

Now, due to the density of $H_{p}^{2}(0,2 \pi)$ in $L^{2}(0,2 \pi)$ the proof is complete.

Acknowledgements. The first author would like to thank the Mathematical Engineering Department of the Universidad de Chile for its hospitality while this work was completed and FONDECYT grants 1000543 and 7000543 for the financial support. The authors are grateful with the referee for his comments which improved the presentation of this work.

\section{REFERENCES}

[1] M. Abdelouhab, J.L. Bona, M. Felland and J.-C. Saut, Nonlocal models for nonlinear, dispersive waves. Physica D 40 (1989) 360-392.

[2] M.J. Ablowitz and A.S. Fokas, The inverse scattering transform for the Benjamin-Ono equation-a pivot to multidimensional problems. Stud. Appl. Math. 68 (1983) 1-10.

[3] T.B. Benjamin, Internal waves of permanent form in fluids of great depth. J. Fluid Mech. 29 (1967) 559-592.

[4] J. Bona and R. Winther, The Korteweg-de Vries equation, posed in a quarter-plane. SIAM J. Math. Anal. 14 (1983) $1056-1106$.

[5] J. Bourgain, Fourier transform restriction phenomena for certain lattice subsets and applications to nonlinear evolution equations. Geom. Funct. Anal. 3 (1993) 107-156, 209-262.

[6] K.M. Case, Benjamin-Ono related equations and their solutions. Proc. Nat. Acad. Sci. USA 76 (1979) 1-3.

[7] T. Cazenave and A. Haraux, An Introduction to Semilinear Evolution Equation. Oxford Sci. Publ. (1998).

[8] J. Colliander and C.E. Kenig, The generalized Korteweg-de Vries equation on the half line. Comm. Partial Differential Equations 27 (2002) 2187-2266.

[9] K.D. Danov and M.S. Ruderman, Nonlinear waves on shallow water in the presence of a horizontal magnetic field. Fluid Dynamics 18 (1983) 751-756.

[10] A.E. Ingham, A further note on trigonometrical inequalities. Proc. Cambridge Philos. Soc. 46 (1950) 535-537.

[11] R. Iorio, On the Cauchy problem for the Benjamin-Ono equation. Comm. Partial Differentiel Equations 11 (1986) 1031-1081.

[12] Y. Ishimori, Solitons in a one-dimensional Lennard/Mhy Jones lattice. Progr. Theoret. Phys. 68 (1982) 402-410.

[13] C.E. Kenig and K. Koenig, On the local well-posedness of the Benjamin-Ono and modified Benjamin-Ono equations. Math. Res. Lett. 10 (2003) 879-895.

[14] C.E. Kenig, G. Ponce and L. Vega, A bilinear estimate with application to the KdV equation. J. Amer. Math Soc. 9 (1996) 573-603.

[15] H. Koch and N. Tzvetkov, On the local well-posedness of the Benjamin-Ono equation in $H^{s}(\mathbb{R})$. Int. Math. Res. Not. 26 (2003) 1449-1464.

[16] Y. Matsuno and D.J. Kaup, Initial value problem of the linearized Benjamin-Ono equation and its applications. J. Math. Phys. 38 (1997) 5198-5224.

[17] S. Micu, On the controllability of the linearized Benjamin-Bona-Mahony equation. SIAM J. Control Optim. 39 (2001) $1677-1696$

[18] H. Ono, Algebraic solitary waves in stratified fluids. J. Phys. Soc. Japan 39 (1975) 1082-1091.

[19] A. Pazy. Semigroups of linear operators and applications to partial differential equations. Springer-Verlag, New York, Appl. Math. Sci. 44 (1983).

[20] G. Perla-Menzala, F. Vasconcellos and E. Zuazua. Stabilization of the Korteweg-de Vries equation with localized damping. Quart. Appl. Math. 60 (2002) 111-129.

[21] G. Ponce, On the global well-posedness of the Benjamin-Ono equation. Diff. Integral Equations 4 (1991) 527-542.

[22] L. Rosier, Exact boundary controllability for the Korteweg-de Vries equation on a bounded domain. ESAIM: COCV 2 (1997) $33-55$.

[23] D.L. Russell and B.-Y. Zhang, Controllability and stabilizability of the third order linear dispersion equation on a periodic domain. SIAM J. Cont. Optim. 31 (1993) 659-676.

[24] D.L. Russell and B.-Y. Zhang, Exact controllability and stabilizability of the Korteweg-de Vries equation. Trans. Amer. Math. Soc. 348 (1996) 3643-3672.

[25] T. Tao, Global well-posedness of the Benjamin-Ono equation in $H^{1}(\mathbb{R})$, preprint (2003). 